\title{
Virtual Non-Contrast Computer Tomography (CT) with Spectral CT as an Alternative to Conventional Unenhanced CT in the Assessment of Gastric Cancer
}

\author{
Shi-Feng Tian, Ai-Lian Liu*, He-Qing Wang, Jing-Hong Liu, Mei-Yu Sun, Yi- \\ Jun Liu
}

\begin{abstract}
Objective: The purpose of this study was to evaluate computed tomography (CT) virtual non-contrast (VNC) spectral imaging for gastric carcinoma. Materials and Methods: Fifty-two patients with histologically proven gastric carcinomas underwent gemstone spectral imaging (GSI) including non-contrast and contrast-enhanced hepatic arterial, portal venous, and equilibrium phase acquisitions prior to surgery. VNC arterial phase (VNCa), VNC venous phase (VNCv), and VNC equilibrium phase (VNCe) images were obtained by subtracting iodine from iodine/water images. Images were analyzed with respect to image quality, gastric carcinoma-intragastric water contrast-to-noise ratio (CNR), gastric carcinoma-perigastric fat $\mathrm{CNR}$, serosal invasion, and enlarged lymph nodes around the lesions. Results: Carcinoma-water CNR values were significantly higher in VNCa, VNCv, and VNCe images than in normal CT images $(2.72,2.60,2.61$, respectively, vs 2.35, p $\leq 0.008)$. Carcinomaperigastric fat CNR values were significantly lower in VNCa, VNCv, and VNCe images than in normal CT images (7.63, 7.49,7.32, respectively, vs $8.48, p<0.001)$. There were no significant differences of carcinoma-water CNR and carcinoma-perigastric fat CNR among VNCa, VNCv, and VNCe images. There was no difference in the determination of invasion or enlarged lymph nodes between normal CT and VNCa images. Conclusions: VNC arterial phase images may be a surrogate for conventional non-contrast CT images in gastric carcinoma evaluation.
\end{abstract}

Keywords: Gastric carcinoma - gemstone spectral imaging - virtual non-contrast - contrast-to-noise ratio - perigastric fat

Asian Pac J Cancer Prev, 16 (6), 2521-2526

\section{Introduction}

Gastric cancer accounts for approximately $8 \%$ of new cancers worldwide (Jemal et al., 2011). Early diagnosis and accurate preoperative staging are critical for improving outcomes, and this includes the identification of the tumor location, depth of invasion, lymph node status, and the presence of metastasis (Monig et al., 2002; Chen et al., 2007; Makino et al., 2011). Current methods for the detection of gastric cancer include upper gastrointestinal endoscopy and barium studies, computed tomography (CT), and magnetic resonance imaging (MRI) (Liu and Chen, 2012). CT imaging is an important means for staging gastric cancer, and contrast-enhanced CT can assess the depth of tumor invasion and lymph node and distant organ metastasis, which may guide gastric cancer staging before surgery and aid postoperative follow-up (Monig et al., 2002; Chen et al., 2007; Makino et al., 2011; Liu and Chen, 2012).

CT imaging spectroscopy can obtain a water/iodine density map through paired substance isolation (Johnson et al., 2007; Graser et al., 2009; Yu et al., 2009; Lin et al., 2011). The water/iodine density map does not contain the iodine component, and this allows the virtual 'subtraction' of the iodine content from a contrast-enhanced, dualenergy CT scan to produce a virtual non-contrast (VNC) image (Li et al., 2012; Liu and Shen, 2012). VNC scan images can be used as a replacement for true non-contrast scans (Toepker et al., 2012). This can eliminate the need for performing both contrast-enhanced and non-contrast scanning. VNC scanning has been examined for use in liver disease, renal disease, and brain hemorrhage (Silva et al., 2011; Tijssen et al., 2014). However, few studies have examined the use of VNC CT scanning for gastric cancer (Pan et al., 2013).

In our institution, the routine and standard protocol for all detail abdominal CT examination comprised spectral imaging of non-contrast and contrast-enhanced hepatic arterial, portal venous, and equilibrium phase acquisitions. The purpose of this study was to compare VNC images with those of conventional $\mathrm{CT}$ for the diagnosis and evaluation of gastric carcinoma. 


\section{Materials and Methods}

\section{Patients}

This study included 52 patients with pathologically confirmed gastric cancer who underwent surgery at our hospital from January 2012 to December 2012. There were 41 males and 11 females with an average age of $65 \pm 10$ years (range, 39 to 89 years). The lesions were located at the fundus and cardia in seven cases, at the gastric body in nine cases, and at the gastric antrum in 36 cases. Postoperative pathological examination confirmed adenocarcinoma in 40 cases, mucous cell carcinoma in four cases, signet ring cell carcinoma in six cases, and squamous cell carcinoma in two cases.

Prior to surgery, all patients underwent gemstone spectral imaging (GSI) including non-contrast and contrast-enhanced hepatic arterial, portal venous, and equilibrium phase acquisitions. This is the standard scanning protocol at our institution, and we believe that it provides superior lesion identification and detection of metastases as compared to other protocols. The triphasic GSI dataset was sent to the workstation, and three sets of VNC images (VNC arterial phase (VNCa), VNC venous phase (VNCv, VNC equilibrium phase (VNCe)) were obtained by subtracting iodine from iodine/water images in the triphasic enhanced GSI images. This study was approved by the Institutional Review Board of the hospital, and all patients provided written informed consent.

\section{Scanning and image processing}

Drugs containing heavy metals were prohibited within 3 days before the scan, and patients were not allowed to eat or drink for 6 to 12 hours before scanning. Patients were asked to drink 800 to $1000 \mathrm{~mL}$ of water 15 to 30 minutes before the scan, and they drank 200 to $300 \mathrm{~mL}$ of water during scanning to fill the stomach and obtain a hypotonic effect. Single breath-hold helical scans were performed with a GE Discovery CT 750 HD scanner (GE Healthcare, USA). The scan range was from the top of the diaphragm to the pubic symphysis. Conventional scanning was performed first, then the GSI mode was used for the contrast-enhanced scan. A high-pressure injector was used to inject the contrast agent (Ioversol; $320 \mathrm{mg} \mathrm{I} / \mathrm{mL}$ ) via the ulnar vein at a flow rate of $2.0-2.5 \mathrm{~mL} / \mathrm{s}$ at a dosage of $1.0 \mathrm{mg} / \mathrm{kg}$ body weight. Arterial phase, venous phase, and equilibrium phase contrast-enhanced images were obtained 28, 55, and $120 \mathrm{~s}$, respectively, after contrast agent injection.

Image analysis and measurements were carried out at an AW4.5 workstation (GE Healthcare). The image reconstruction methods for the conventional and dual energy images were done by adaptive statistical iterative reconstruction techniques. Corresponding VNC images were generated through iodine-water pair substance isolation of the contrast-enhanced images in the three phases (Figure 1). Conventional scan images and $\mathrm{VNCa}, \mathrm{VNCV}$, and VNCe images were analyzed from the perspectives of image quality, gastric carcinomaintragastric water contrast-to-noise ratio (CNR), gastric carcinoma-perigastric fat CNR, serosal invasion, and enlarged lymph nodes around the lesions.

\section{Image quality}

Two radiologists with more than 30 years of experience (observer No. 1 and No. 2) subjectively assessed the image quality of the conventional unenhanced images and VNC images in the 3 phases. Based on the severity of image artifacts (whether linear high-density artifacts were present in the renal pelvis), the clarity of anatomical details and its impact on diagnosis were scored on a five-point scale (Behrendt et al., 2009). The specific scoring criteria were as follows: 5 points (excellent), anatomical structures and details are clear and there is no obvious noise or artifacts; 4 points (good), anatomical structures and details are less clear, and noise and artifacts are increased; 3 points (moderate :, most of the anatomic structures are clear, the details are less clear, and noise and artifacts are obvious, but still acceptable; 2 points (poor), anatomical structures are unclear, it is difficult to identify details, and noise and artifacts are very obvious; 1 (very poor), anatomical
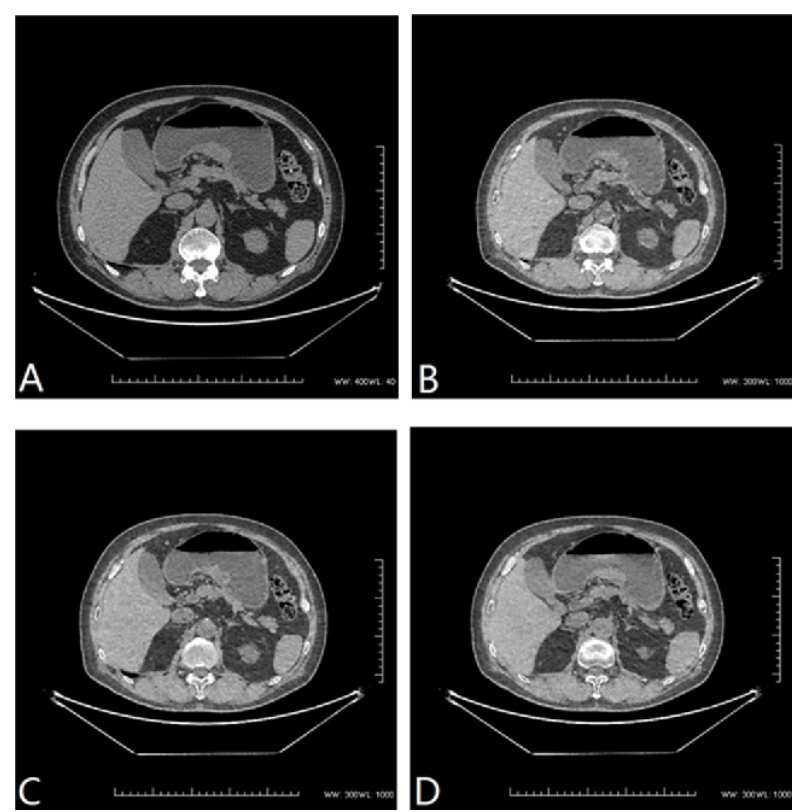

Figure 1. Conventional Plain Scan Image (A), Arterial Phase Virtual Non-contrast (VNC) Image (B), Venous Phase VNC Image (C), Equilibrium Phase VNC Image (D)
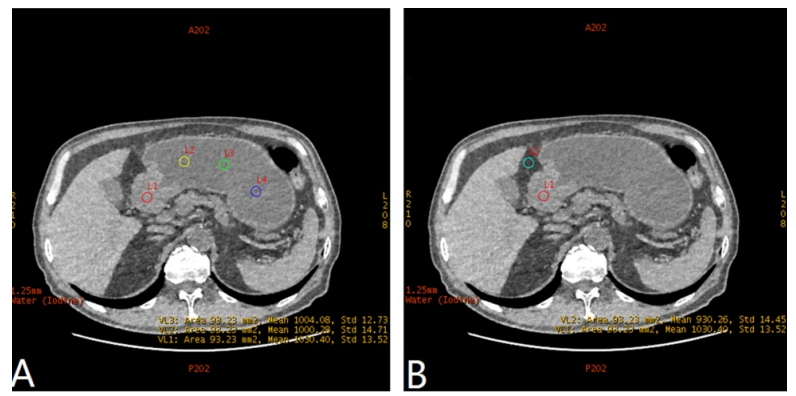

Figure 2. Arterial Phase Virtual Non-contrast (VNC) Images. A) A region of interest (ROI) was placed at the lesion, and three ROIs were placed evenly in the intragastric water for measurement of the carcinoma-intragastric water contrast-tonoise (CNR). B) For measurement of the carcinoma-perigastric fat CNR, a ROI was placed at the lesion and at the perigastric fat. 
structures are blurred, details cannot be identified, and noise and artifacts are very obvious. Images with 3 points or more were considered to meet the requirements for diagnosis. The consistency between the assessment results of the two radiologists with respect to image quality was analyzed by determination of the Kappa value.

\section{Carcinoma-intragastric water and carcinoma-perigastric fat $C N R$}

The carcinoma-intragastric water and carcinomaperigastric fat CNRs were independently measured by a senior radiologist with 25 years of CT diagnosis experience who was unaware of the results of the image quality analysis (observer No. 3). The specific method was as follows. A region of interest (ROI) was placed at the lesion, avoiding necrotic areas as much as possible. Three ROIs were evenly placed in the gastric water, avoiding the artifact area caused by the gas-liquid plane junction. An ROI was placed at a location of clear perigastric fat (Figure 2). The location, shape, and size of the ROIs were kept consistent in the four groups (conventional CT, $\mathrm{VNCa}, \mathrm{VNCv}, \mathrm{VNCe}$ ). That is, contrast enhanced images were used to identify the location of the tumor, and then the ROI's were placed in the corresponding area of the unenhanced scan. The area of the ROI in the intragastric water was approximately $1.0 \mathrm{~cm}^{2}$, and the area of the ROI in the perigastric fat was approximately $0.5 \mathrm{~cm}^{2}$. If area of the lesion or the perigastric fat was small, the ROI area was adjusted so that it did not exceed the range of lesion or perigastric fat. As compared with $\mathrm{VNCv}$ and $\mathrm{VNCe}$, which both had more homogeneous intensified images, VNCa generally shows inhomogeneous intensified images among gastric carcinoma patients.

The clarity of CT images (i.e., spatial resolution and the contrast resolution) reflecting a lesion can be analyzed objectively using the CNR. The CNR represents the contrast between two tissues (normal and pathological) in the image. The lower image noise, the better the lesion display and image quality. CNR is a ratio and does not have a unit.

CNR was calculated according to the following formula (Ye et al., 2011):

$\mathrm{CNR}=($ Mean 1-Mean 2) $/$ Noise.

For conventional unenhanced images, Mean 1 was the average $\mathrm{CT}$ value of the lesion ROI, Mean 2 was the average CT value of the intragastric water or the perigastric fat ROI, and Noise was the standard deviation of the intragastric water or perigastric fat ROI. For VNC images, Mean 1 was the average water base value of the lesion ROI, Mean 2 was the average water baseline value of the intragastric water or perigastric fat ROI, and Noise was the standard deviation of the intragastric water or perigastric fat ROI.

\section{Serosal invasion and perigastric lymph node enlargement}

The surgical records of the 52 patients were reviewed, and pathologically diagnosed serosal invasion was used as the standard to calculate the accuracy of the images for identification of serosal invasion in the four groups. Serosal invasion (T3 vs T4a) was chosen as the measure of accuracy as opposed to adjacent organ invasion (T4a vs T4b) because serosal invasion occurs earlier than adjacent organ invasion. A fuzzy perigastric fat gap at the lesion was considered to indicate serosal invasion. Lymph node enlargement around the lesions in conventional unenhanced images was used as the standard to calculate the accuracy of VNC images for the diagnosis of lymph node enlargement around the lesions in the three VNC groups. Serosal invasion and perigastric lymph node enlargement were evaluated by the same observer (No. 3).

\section{Statistical analysis}

Continuous data were presented as means and standard deviations (SDs), and categorical data as counts. The Kappa value was calculated to assess inter-rater reliability. A Kappa value of 0.01-0.20 indicates slight agreement; 0.21-0.40 indicates fair agreement; 0.41-0.60 indicates moderate agreement; 0.61-0.80 indicates substantial agreement; 0.81-0.99 indicates almost perfect agreement. Paired-t test was performed to compare the differences between normal CT versus VNC in scores, carcinomawater CNR, and carcinoma-perigastric fat CNR. The McNemar test was applied to examine the consistency of invasiveness and lymph nodes growth between normal CT versus different VNC phases. Statistical analysis was considered significant when a two-sided p-value was $<0.05$. All statistical analyses were performed with SPSS 17.0 statistics software (SPSS Inc., Chicago, IL).

\section{Results}

The assessment of agreement between the two observers showed substantial reliability for normal CT, VNCa, VNCv, and VNCe scores with kappa values of $0.795,0.808,0.846$, and 0.877 , respectively. As the analysis showed substantial to almost perfect agreement, the scores of observer No. 2 were used for the analysis.

Comparisons of image quality score, carcinoma-water $\mathrm{CNR}$, and carcinoma-perigastric fat CNR between normal CT and different VNC phases are shown in Table 1. The

Table 1. Image Quality Scores, Carcinoma-water CNR, and Carcinoma-perigastric Fat CNR between Normal and Different VNC Phases

\begin{tabular}{lcccc}
\hline \multicolumn{2}{c}{ Conventional non-contrast CT } & \multicolumn{2}{c}{ VNC } \\
\cline { 3 - 5 } & & $\begin{array}{c}\text { Arterial phase } \\
\text { (VNCa) }\end{array}$ & $\begin{array}{c}\text { Venous phase } \\
\text { (VNCv) }\end{array}$ & $\begin{array}{c}\text { Equilibrium phase } \\
\text { (VNCe) }\end{array}$ \\
\hline Image quality scores & $4.73 \pm 0.45$ & $4.12 \pm 0.5^{*}$ & $3.44 \pm 0.54^{* \dagger}$ & $3.38 \pm 0.49^{* \dagger}$ \\
Carcinoma-water CNR & $2.35 \pm 0.39$ & $2.72 \pm 0.69^{*}$ & $2.6 \pm 0.64^{*}$ & $2.61 \pm 0.68^{*}$ \\
Carcinoma-perigastric fat CNR & $8.48 \pm 1.23$ & $7.63 \pm 1.17^{*}$ & $7.49 \pm 1.52^{*}$ & $7.32 \pm 1.27^{*}$ \\
\hline
\end{tabular}

CNR, contrast-to-noise ratio; $\mathrm{CT}$, computed tomography; VNC, virtual non-contrast; $*$ Significant difference as compared with normal CT; ${ }^{\dagger}$ Significant difference as compared with VNC arterial phase 


\begin{tabular}{llrc}
\hline & & \multicolumn{2}{c}{ Conventional non-contrast CT } \\
\cline { 3 - 4 } VNC arterial phase (VNCa) & Invasion & No-invasion \\
& Invasion & 23 & 1 \\
VNC venous phase (VNCv) & No invasion & 0 & 28 \\
& Invasion & 21 & 11 \\
VNC equilibrium phase (VNCe) & No invasion & 2 & 18 \\
& Invasion & 23 & 0.022 \\
\hline
\end{tabular}

Supplemental Table 1. Consistency of Lymph Node Enlargement between Normal CT and VNC

\begin{tabular}{|c|c|c|c|c|}
\hline & & \multicolumn{3}{|c|}{ Conventional non-contrast CT } \\
\hline & & Enlargement & No enlargement & p-value \\
\hline \multirow[t]{2}{*}{ VNC arterial phase (VNCa) } & Enlargement & 40 & 0 & 1 \\
\hline & No enlargement & 0 & 12 & \\
\hline \multirow[t]{2}{*}{ VNC venous phase (VNCv) } & Enlargement & 40 & 0 & 1 \\
\hline & No enlargement & 0 & 12 & \\
\hline \multirow[t]{2}{*}{ VNC equilibrium phase (VNCe) } & Enlargement & 40 & 0 & 1 \\
\hline & No enlargement & 0 & 12 & \\
\hline
\end{tabular}

mean image quality scores were significantly lower in $\mathrm{VNCa}, \mathrm{VNCv}$, and VNCe images as compared to that of normal CT images $(4.12 \pm 0.5,3.44 \pm 0.54,3.380 .49$, respectively, vs $4.73 \pm 0.45, \mathrm{p}<0.001)$. $\mathrm{VNCv}$ and $\mathrm{VNCe}$ images also exhibited significantly lower image quality scores in comparison with VNCa images (3.44 \pm 0.54 , $3.38 \pm 0.49$, respectively $v s 4.12 \pm 0.5, \mathrm{p}<0.001)$. The mean carcinoma-water CNR values were significantly higher in $\mathrm{VNCa}, \mathrm{VNCv}$, and $\mathrm{VNCe}$ images in comparison with the value in normal CT images $(2.72 \pm 0.69,2.60 \pm 0.64$, 2.610 .68 , respectively, vs $2.35 \pm 0.39$, $\mathrm{p} \leq 0.008$ ). The mean carcinoma-perigastric fat CNR values were significantly lower in $\mathrm{VNCa}, \mathrm{VNCv}$, and $\mathrm{VNCe}$ images in comparison with that of normal CT images $(7.63 \pm 1.17,7.49 \pm 1.52$, $7.32 \pm 1.27$, respectively, vs $8.48 \pm 1.23, \mathrm{p}<0.001)$. There were no significant differences of carcinoma-water CNR and carcinoma-perigastric fat $\mathrm{CNR}$ among $\mathrm{VNCa}, \mathrm{VNCV}$, and VNCe images (Table 1).

Identification of invasion with normal CT versus different VNC phases is shown in Table 2. There was no significant difference in the determination of invasion between normal CT and VNCa images $(p=1.000)$. As compared with normal CT images, invasion was significantly different as determined by $\operatorname{VNCv}(\mathrm{p}=0.022)$ and $\mathrm{VNCe}(\mathrm{p}<0.001)$ images, respectively.

The determination of lymph node enlargement was similar in normal CT versus $\mathrm{VNCa}, \mathrm{VNCv}$, and $\mathrm{VNCe}$ images $(\mathrm{p}=1.000)$ (Table 3$)$.

\section{Discussion}

The purpose of this study was to examine the utility of VNC spectral imaging for the identification of gastric carcinoma and determination of lymph node metastasis and the results showed that arterial phase VNC images are equivalent to those of conventional CT images for the identification of gastric carcinoma and assessment of lymph node metastasis. There were, however, differences between conventional $\mathrm{CT}$ and VNC venous and equilibrium phase scans, and caution should be taken when VNC images are used to asses gastric serosal invasion.
CT spectroscopy isolates iodine, and obtains the water/ iodine density map under the conditions of a contrastenhanced scan. The water/iodine density map does not contain an iodine component, thereby producing a VNC scan. In VNC scan images, the water/iodine density of the ROI can be measured as the water content per unit volume (a concentration value with a unit of $\mathrm{mg} / \mathrm{mL}$ ). The VNC scan is obtained through post-processing of contrastenhanced scan images; therefore the image corresponds to the contrast-enhanced image at each pixel. Deviation of the ROI position that occurs during the dynamic scan process can be reduced to a maximum extent, and the data error can be reduced while shortening the examination time. These advantages are especially beneficial for pediatric patients, those with movement disorders, and in cases where emergency imaging is necessary (Jiang and Han, 2010). As VNC scanning allows omission of the conventional non-enhanced scan, the radiation dose of CT scanning is reduced, an important area of current research (Zhang et al., 2010). Ma et al. (2012) reported that the radiation dose during a single phase, and the total radiation dose of the VNC scan were reduced compared with those of conventional scan. Mileto et al. (2012) pointed out that VNC scanning can reduce the radiation dose received by patients by approximately $26.7 \%$ due to the omission of the conventional scan.

VNC CT has been shown to produce attenuation values similar to those of single-energy non-contrast images (Toepker et al., 2012). The technique has been studied in cases of renal and urinary tract disorders (Graser et al., 2009; Song et al, 2011; Lundin et al., 2012), brain hemorrhage (Tijssen et al., 2014), and abdominal imaging (Silva et al., 2011; Mileto et al., 2012; Yu et al., 2013), and generally has been shown to produce images consistent with conventional non-contrast CT images. Few studies, however, have examined VNC CT with respect to gastric cancer. Chen et al. (2013) reported that CT imaging spectroscopy was useful for assessing the degree of differentiation of gastric cancer. Pan et al. (2013) examined 96 patients with suspected gastric cancer with dual-energy spectral CT. Patients received 
dual-phasic scans (arterial and venous), and material decompensation images were constructed. The results showed an improvement of N-staging accuracy with the material decompensation images.

The clarity of gastric carcinoma CT images can be objectively analyzed using the image noise and the CNR (Yamada et al., 2014). The lower the image noise, the higher the CNR. A higher CNR is associated with sharper display of the lesion, and better image quality. The results of this study showed that the carcinoma-intragastric water $\mathrm{CNR}$ in the VNC scan images was higher than that in the conventional plain scans. A possible reason is that when the VNC scan images were obtained through water/iodine isolation of the contrast-enhanced images, iodine was not isolated completely resulting in VNC scan images that were similar to slightly enhanced images. In the venous and equilibrium phase VNC scan images, the carcinomaintragastric water CNR was not different from that of the conventional scan, indicating that during gastric filling the VNC scan images in the two phases can achieve effects similar to that of conventional scanning. On the other hand, the gastric carcinoma-perigastric fat CNRs of the VNC scan images in the three phases were lower than that of the conventional scan images. This is possibly due to perigastric fat displayed during water/iodine isolation. Therefore, caution should be taken when VNC images are used to assess serosal invasion.

Different noise levels of the resulting images are likely related to the residual iodine attenuation in the VNC images. Incomplete elimination of the iodine attenuation is a well-known fact that directly relates to the performance of the dual-energy-scanner. Reduction of image noise will theoretically improve the quality of VNC images. Study is ongoing regarding the use of adaptive statistical iterative reconstruction which may effectively reduce image noise (Fang and Li, 2013), thereby further enhancing the CNR and quality of VNC images. This improvement, however, still requires further in-depth study.

Linear high-density artifacts were found in the renal pelvis and ureter in the parenchymal phase in VNC images. We speculate that the reason for this is that a large amount of contrast agent is excreted via the renal pelvis in the parenchymal phase, resulting in a high concentration of iodine in renal pelvis and ureter that exceeds the threshold of iodine removal during water/iodine isolation. Therefore, the high-concentration iodine in the pelvis and ureter could not be differentiated.

There was no difference in the display of enlarged lymph nodes between VNC images and conventional scan images, indicating that VNC images can be used to assess perigastric lymph node metastasis. Other study has shown that VNC images are useful for evaluating mediastinal lymph nodes (Yoo et al., 2013).

This study has some limitations that should be considered. Only primary gastric lesions, local serosal invasion, and adjacent lymph node metastasis were examined. We did not evaluate the value of VNC images for assessing liver metastasis or distant organ metastasis, and did not characterize patients in terms of pTNM staging. Our goal was to determine if VNC images could replace conventional unenhanced $\mathrm{CT}$ images in the assessment of gastric cancer; we did not focus specifically on dual-energy spectral CT imaging.

In conclusions, CT imaging spectroscopy arterial phase VNC images can be an alternative to conventional unenhanced CT images for the display of gastric cancer lesions and assessment of lymph node metastasis. Caution should be taken when they are used to assess gastric serosal invasion. The application of VNC scan requires one less conventional CT scan, which will lower the radiation dose received by patients and shorten the examination time. Further study of the technique for the assessment of gastric cancer is warranted.

\section{References}

Behrendt FF, Schmidt B, Plumhans C, et al (2009). Image fusion in dual energy computed tomography: effect on contrast enhancement, signal-to-noise ratio and image quality in computed tomography angiography. Invest Radiol, 44, 1-6.

Chen CY, Hsu JS, Wu DC, et al (2007). Gastric cancer: preoperative local staging with $3 \mathrm{D}$ multi-detector row CT--correlation with surgical and histopathologic results. Radiology, 242, 472-82.

Chen L, Xue Y, Duan Q, et al (2013). Assessing the degree of differentiation of gastric cancer using CT imaging spectroscopy. Chinese Med Imaging Technol, 29, 225-9.

Fang L, Li L (2013). Application progress of adaptive statistical iterative reconstruction in reducing radiation dose. $C T$ Theory and Applications, 22, 207-13.

Graser A, Johnson TR, Hecht EM, et al (2009). Dual-energy $\mathrm{CT}$ in patients suspected of having renal masses: can virtual nonenhanced images replace true nonenhanced images? Radiology, 252, 433-40.

Jemal A, Bray F, Center MM, et al (2011). Global cancer statistics. CA Cancer J Clin, 61, 69-90.

Jiang J, Han D (2010). Clinical application and advancement of dual-energy CT virtual non-contrast scan. J Radiol, 46, 958-60.

Johnson TR, Krauss B, Sedlmair M, et al (2007). Material differentiation by dual energy CT: initial experience. Eur Radiol, 17, 1510-7.

Li C, Zhang S, Zhang H, et al (2012). Using the K-nearest neighbor algorithm for the classification of lymph node metastasis in gastric cancer. Comput Math Methods Med, 2012, 876545.

Lin X, Shen Y, Chen K (2011). Basic principle of CT imaging spectroscopy and research progress in clinical application. Chinese J Radiol, 45, 798-800.

Liu A, Shen Y (2012). In "CT spectroscopy spectrum for clinical application”, Ed People's Military Medical Publishing House, Beijing pp 18-19.

Liu Y, Chen K (2012). Diagnostic radiology status and progress of early gastric cancer. Modern Digestion Invasive Diagnosis, 17, 37-8.

Lundin M, Liden M, Magnuson A, et al (2012). Virtual noncontrast dual-energy CT compared to single-energy CT of the urinary tract: a prospective study. Acta Radiol, 53, 689-94.

Ma ZP, Zhou JJ, Liu XL, et al (2012). Diagnostic value of dualenergy CT dual-phase contrast-enhanced scan combined with virtual non-contrast scan for renal clear cell carcinoma. Chinese J Radiol, 46, 687-92.

Makino T, Fujiwara Y, Takiguchi S, et al (2011). Preoperative $\mathrm{T}$ staging of gastric cancer by multi-detector row computed tomography. Surgery, 149, 672-9.

Mileto A, Mazziotti S, Gaeta M, et al (2012). Pancreatic dualsource dual-energy CT: is it time to discard unenhanced 
imaging? Clin Radiol, 67, 334-9.

Monig SP, Schroder W, Baldus SE, et al (2002). Preoperative lymph-node staging in gastrointestinal cancer-correlation between size and tumor stage. Onkologie, 25, 342-4.

Pan Z, Pang L, Ding B, et al (2013). Gastric cancer staging with dual energy spectral CT imaging. PLoS One, $8,53651$.

Silva AC, Morse BG, Hara AK, et al (2011). Dual-energy (spectral) CT: applications in abdominal imaging. Radiographics, 31, 1031-46; discussion 1047-50.

Song KD, Kim CK, Park BK, et al (2011). Utility of iodine overlay technique and virtual unenhanced images for the characterization of renal masses by dual-energy CT. Am J Roentgenol, 197, 1076-82.

Tijssen MP, Hofman PA, Stadler AA, et al (2014). The role of dual energy CT in differentiating between brain haemorrhage and contrast medium after mechanical revascularisation in acute ischaemic stroke. Eur Radiol, 24, 834-40.

Toepker M, Moritz T, Krauss B, et al (2012). Virtual non-contrast in second-generation, dual-energy computed tomography: reliability of attenuation values. Eur J Radiol, 81, 398-405.

Yamada Y, Jinzaki M, Tanami Y, et al (2014). Virtual monochromatic spectral imaging for the evaluation of hypovascular hepatic metastases: the optimal monochromatic level with fast kilovoltage switching dual-energy computed tomography. Invest Radiol, 47, 292-8.

Ye XH, Zhou C, Wu GG, et al (2011). Effects of CT spectroscopy monoergic imaging for the detection of different liver tumors: A preliminary study. Chinese J Radiol, 45, 718-22.

Yoo SY, Kim Y, Cho HH, et al (2013). Dual-energy CT in the assessment of mediastinal lymph nodes: comparative study of virtual non-contrast and true non-contrast images. Korean J Radiol, 14, 532-9.

Yu L, Primak AN, Liu X, et al (2009). Image quality optimization and evaluation of linearly mixed images in dual-source, dual-energy CT. Med Phys, 36, 1019-24.

Yu Y, Lin X, Chen K, et al (2013). Hepatocellular carcinoma and focal nodular hyperplasia of the liver: differentiation with CT spectral imaging. Eur Radiol, 23, 1660-8.

Zhang Y, Ma G, Wang W et al (2010). Strategies to reduce radiation dose of spiral CT. Intern J Radiat Med, 33, 139-43. 